\title{
Deutschland: ein Land der Mieter? Die Rolle von Erwartungen über zukünftige Immobilienpreisentwicklungen
}

\author{
Niklas Gohl • Peter Haan • Claus Michelsen · Felix Weinhardt
}

Online publiziert: 18. Oktober 2019

(C) Der/die Autor(en) 2019

Zusammenfassung Mehr als die Hälfte aller Haushalte in Deutschland wohnen zur Miete - ein im internationalen Vergleich sehr hoher Wert. Bisherige Studien haben vor allen Dingen den regulatorischen Rahmen des Immobilienmarkts hervorgehoben, der Mietwohnungen in Deutschland systematisch begünstigt. Allerdings gibt es keine Studien, die diese Erklärungen empirisch eindeutig untermauern können: einige Arbeiten betonen die Bedeutung von fundamentalen Marktdaten wie die Einkommensentwicklung, demographische Faktoren oder den Zuzug in eine Region. Diese Studie greift einen Aspekt auf, der bislang nicht in der Literatur diskutiert wurde. Untersucht wird, ob und zu welchem Anteil die geringe Eigentümerquote in Deutschland durch pessimistische Erwartungen bezüglich zukünftiger Immobilienpreisentwicklungen erklärt werden kann - erwarten Mieter keine oder nur geringe Preissteigerungen, so sinkt die Attraktivität einer Investition in die eigenen vier Wände. Für die empirische Analyse werden Daten aus einer neuen, repräsentativen

N. Gohl · P. Haan · C. Michelsen · F. Weinhardt DIW Berlin, Berlin, Deutschland

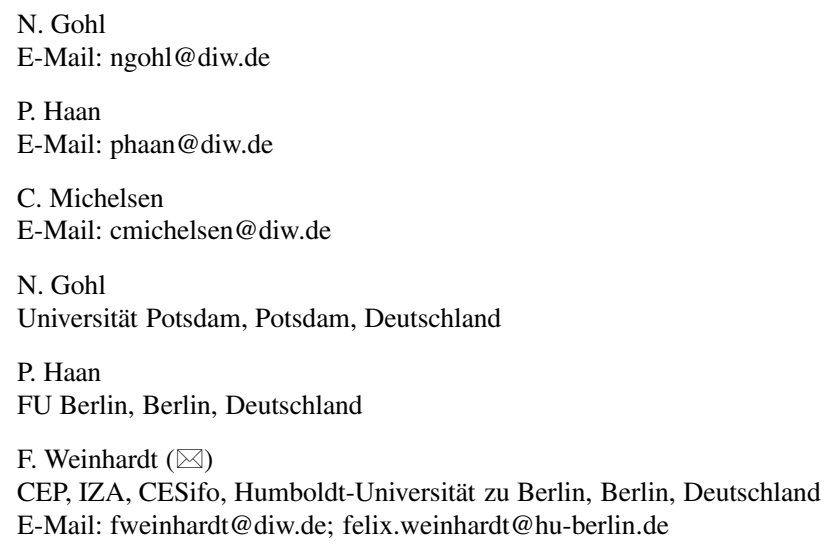


Befragung, erhoben im Rahmen des SOEP-IS, ausgewertet. Befragte ausländischer Herkunft sind optimistischer hinsichtlich der Immobilienpreisentwicklung. Sie erwarten langfristig einen signifikant höheren Preisanstieg. Dies legt nahe, dass die erheblich pessimistischeren Preiserwartungen der einheimischen Bevölkerung auch dazu führen, dass sie sich seltener als die Bevölkerung in anderen Ländern für selbstgenutztes Wohneigentum entscheiden.

Schlüsselwörter Wohneigentumsquote $\cdot$ Preiserwartungen $\cdot$ Immobilieninvestition

\title{
Germany, a country of renters? The role of price expectations
}

\begin{abstract}
More than half of German households are renting - a large proportion in international comparison. Existing studies have examined regulartory constraints and the legal system favouring certain aspects of the rental market. Other studies have examined market fundamentals such as incomes, demographic factors and migration but the overall empirical support for explaining the high proportion of renters, and low owner-occupation in Germany is weak. This study contributes by examining a new factor: pessimistic expectations about future price growth in Germany. In particular, if existing renters expect low price growth they would see little reason to move into owner-occupation. We analyse these questions based on information from the SOEP-IS, a representative household panel dataset. Our main finding is that households of foreign nationality have significantly more positive price expectations. We conclude that pessimistic expectations about future prices are one factor contributing to the low rates of owner-occupation in Germany.
\end{abstract}

Keywords Owner occupation · Price expectations · Investment decisions

\section{Einleitung}

Deutschland ist ein Land der Mieter: in keinem anderen Staat der europäischen Union ist die Wohneigentumsquote geringer als hierzulande. In der aktuellen politischen Debatte wird dies zunehmend als Problem wahrgenommen, denn mit dem Erwerb der eigenen vier Wände werden einige Vorteile verbunden: So wird häufiger argumentiert, Immobilieneigentümer wären im Alter vermögender als Mieter (siehe bspw. Turner und Luea 2009), könnten zu günstigeren Kosten wohnen (siehe bspw. Leifels und Raffelhüschen 2014), wären insgesamt zufriedener mit ihrer Lebenssituation (siehe bspw. Diaz-Serrano 2009), was darüber hinaus auch zu positiven Externalitäten für die Nachbarschaft führt (siehe bspw. Haurin et al. 2002; Rohe und Lindblad 2013).

Trotz historisch niedriger Zinsen verharrt die Wohneigentumsquote auf dem Niveau der Jahrtausendwende und sinkt sogar bei Haushaltsgruppen zwischen 25 und 40 Jahren, die typischerweise erstmals Immobilienvermögen aufbauen (Michelsen 2017). Auf diese Entwicklung hat die Politik reagiert und jüngst das sogenannte „Baukindergeld“ eingeführt, dass jungen Familien den Weg ins Eigenheim ebnen soll (Michelsen et al. 2018). Aber auch über die Wiederbelebung der Bausparför- 
derung wird derzeit diskutiert: Die Wohnungsbauprämie, die bereits im Jahr 1952 eingeführt wurde, existiert zwar noch, hat aber aufgrund der seit mehr als 20 Jahren unveränderten Einkommensgrenzen für Förderberechtigte erheblich an Bedeutung verloren, da die Einkommen zwischenzeitlich erheblich gestiegen sind.

Erklärungsansätze, weshalb sich die Eigentumsquoten in den einzelnen Ländern und in Deutschland im Besonderen so stark unterscheiden, sind vielfältig. So werden in Panelstudien beispielsweise demographische und sozio-ökonomische Gründe für die Erklärung der Wohneigentumsbildung herangezogen. Es zeigt sich, dass beispielsweise die Einkommensentwicklung positiv mit der Eigentumsbildung korreliert ist. Ein großer Anteil der minderjährigen Bevölkerung hingegen dämpft die aggregierte Neigung, Wohneigentum zu bilden. Ein höherer Anteil der Bevölkerung im erwerbsfähigen Alter ist positiv mit der Eigentumsquote korreliert (Fisher und Jaffe 2003; Chiuri und Jappelli 2010; Lerbs und Oberst 2014; Clark et al. 1997).

Einige Studien stellen auch auf die staatlichen Aktivitäten ab. Werden umfangreiche Transfers an Mieterhaushalte gewährt oder ist ein intensiver Mieterschutz gesetzlich garantiert oder ist ein umfangreicher Bestand öffentlicher oder geförderter Wohnungen vorhanden, dann ist die Neigung, Eigentum selbst zu nutzen geringer (Fisher und Jaffe 2003; Holmqvist und Turner 2014; Mulder und Wagner 1998). Zudem gibt es Untersuchungen, die auf das Steuersystem verweisen und anführen, dass Miet- und Eigentumswohnungen häufig steuerlich nicht neutral behandelt werden (Voigtländer 2009). Ähnliches hat auch der Sachverständigenrat zur Gesamtwirtschaftlichen Entwicklung in seinem Jahresgutachten 2015 festgestellt und die Verzerrung der Einkommensbesteuerung zu Ungunsten des Wohneigentums beklagt (SVR Wirtschaft 2016). Zudem gibt es Arbeiten, die auf die Höhe der Grunderwerbsteuer hinweisen. Je höher diese ist, desto teuerer werden Transaktionen von Immobilien und desto unattraktiver deren Erwerb für die eigene Nutzung. Die Liquidität des Marktes leidet unter hohen Transaktionssteuersätzen und damit die Wohneigentumsquote (Voigtländer 2009; Sommer und Sullivan 2018). Zuletzt ist auch auf staatliche Unterstützungsprogramme zu verweisen: dort, wo Wohneigentum stärker gefördert wird, äußert sich dies auch in höheren Eigentumsquoten.

Zahlreiche Arbeiten haben zudem auf die Finanzierungsseite verwiesen und die Effizienz des Kredit- und Bankenwesens als Erklärungsfaktor angeführt. Sind beispielsweise hohe Eigenkapitalanforderung zu erfüllen, um eine Immobilienfinanzierung abzuschließen, dann liegt die Eigentumsquote häufig niedriger als in Ländern mit geringeren Anforderungen (Chiuri und Jappelli 2003).

Trotz dieser vielen bereits untersuchten Faktoren scheinen auch andere Aspekte eine Rolle zu spielen, die mit den genannten Größen nicht erfasst werden. Zumindest deuten darauf die stets signifikanten Effekte der länderspezifischen Kontrollvariablen in Panelregressionen hin. Häufig wird auch argumentiert, dass die Eigentumsrate etwas mit Einstellungen und der Erwartungsbildung zu tun hat: so ist die Wahrscheinlichkeit größer, dass Individuen Wohneigentum bilden, wenn die Eltern dies vorgelebt haben (Henretta 1984). Ein weiterer Kanal ist die Erwartung über die zukünftige Entwicklung von Immobilienpreisen: Herrscht die Überzeugung, dass weitere Preissteigerungen wahrscheinlich sind, dürfte dies die Kaufneigung steigern. Sind die Erwartungen eher pessimistisch, dürfte die Eigentumsbildung eher gebremst werden. Die folgende Untersuchung setzt an diesem Aspekt an und 
Abb. 1 Eigentümerstruktur. (Quelle: SVR Wirtschaft 2016)

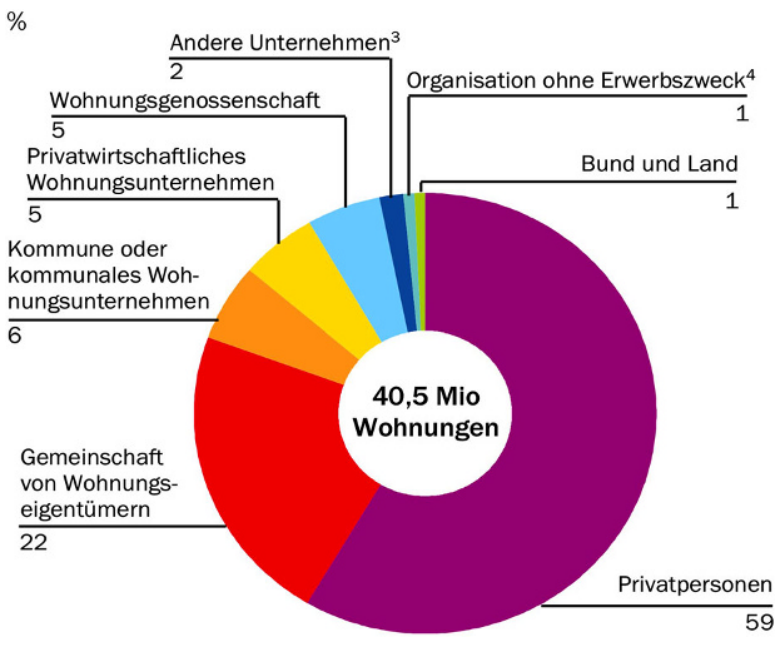

analysiert, inwiefern sich die Erwartungen an die kurz- und mittelfristige Immobilienpreisentwicklung zwischen Individuen mit deutscher Nationalität und denen mit ausländischen Wurzeln unterscheiden. Genutzt wird eine zusätzliche Frage in der Innovationsstichprobe des Sozio-oekonomischen Panels, die in den letzten zwei Wellen erhoben wurde. Der Analyse werden zunächst einige stilisierte Fakten über den Wohnungsmarkt in Deutschland und im internationalen Vergleich vorgeschaltet. Danach wird das empirische Design beschrieben und die Ergebnisse der Analyse vorgestellt. Der abschließende Abschnitt fasst die Erkenntnisse zusammen und setzt diese in den polit-ökonomischen Kontext der aktuellen Wohnungsmarktpolitik.

\section{Wohneigentum in Deutschland und im internationalen Vergleich: stilisierte Fakten}

Der empirischen Analyse wird eine stilisierte Betrachtung der Strukturen auf dem deutschen Immobilienmarkt vorgeschaltet. Dabei werden die Strukturen in Deutschland mit denen in anderen entwickelten Volkswirtschaften verglichen.

\subsection{Eigentumsquote und Eigentümerstruktur}

Der deutsche Wohnungsmarkt ist durch eine im internationalen Vergleich niedrige Wohneigentumsquote geprägt. Weniger als die Hälfte aller Haushalte in Deutschland wohnen in den eigenen vier Wänden. Selbst wenn die Länder des Ostblocks außer Acht gelassen werden - dort gab es einige politische Unterschiede - fällt auf, dass die Eigentumsquote sich erheblich von den direkten Nachbarn unterscheidet. So bewohnen in Frankreich etwa $65 \%$ der Bevölkerung ihre Immobilien selbst, in den Niederlanden knapp 70\% und Belgien knapp 75\%. Nur die Schweiz weist eine noch geringere Eigentumsquote auf als Deutschland (vgl. Ahlfeldt und Maennig in diesem Band). 


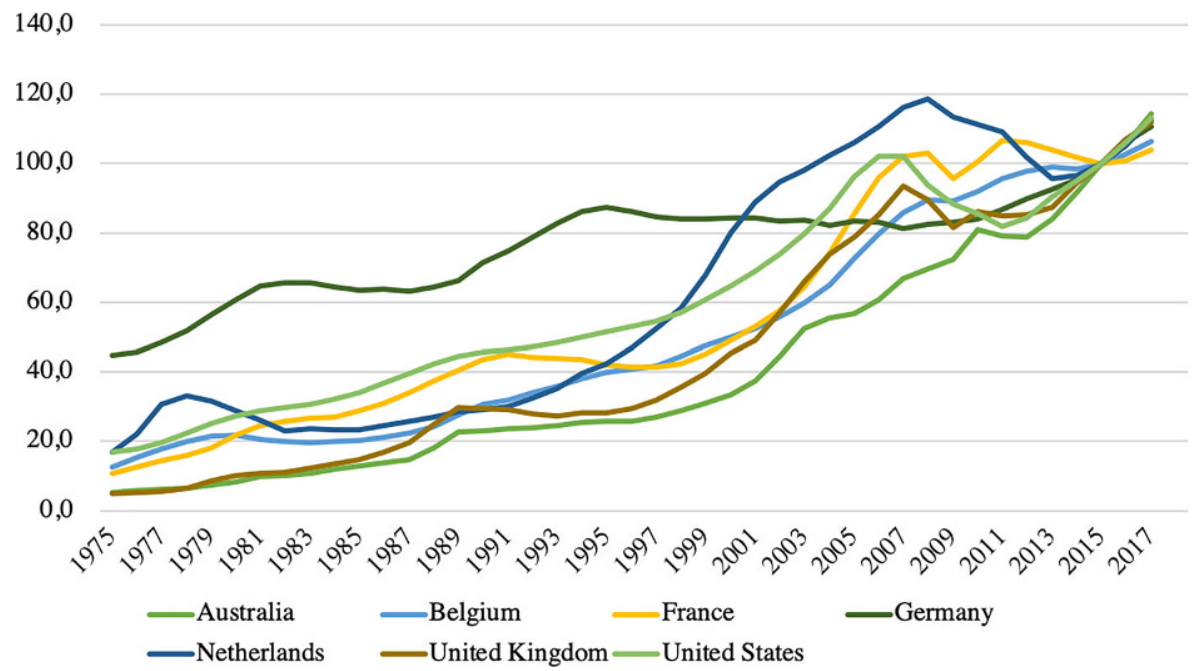

Abb. 2 Immobilienpreise. (Quelle: OECD)

Wichtigste Eigentümergruppe auf dem Wohnungsmarkt sind Privatpersonen und Eigentümergemeinschaften (vgl. SVR Wirtschaft 2016) - zusammen haben diese Gruppen einen Marktanteil von rund $80 \%$. Wohnungsgenossenschaften und kommunale Wohnungsunternehmen halten weitere $11 \%$ des Wohnungsbestands. Alle anderen Eigentümergruppen teilen sich die verbleibenden rund acht Prozent des Markts (vgl. Abb. 1).

\subsection{Immobilienpreise}

In den letzten zehn Jahren sind die Immobilienpreise in Deutschland stark gestiegen. Dies war nicht immer so. Um die Jahrtausendwende und in den Jahren danach stagnierten die Preise für Wohnimmobilien für längere Zeit. Auffällig ist darüber hinaus, dass die Preisentwicklung in Deutschland in der langfristigen Perspektive bei weitem nicht so kräftig verlief wie in anderen Ländern. Zudem ist die globale Immobilienpreisblase - vor allem sichtbar in den USA - scheinbar vollständig am deutschen Wohnungsmarkt vorbeigegangen (Abb. 2).

\subsection{Finanzierung}

Eine in der Literatur intensiv diskutierte Größe ist die Finanzierungsseite von Immobilieninvestitionen: Es besteht ein klarer Zusammenhang zwischen Zugang zu Krediten und der Eigentumsquote. Gemessen wird dies oftmals an Indikatoren wie der maximalen Beleihung (LTV), der Vertragsbindung und der Art der Verzinsung. Gemessen am LTV gehört Deutschland zu den Ländern, in denen es eher schwierig ist, eine Kreditfinanzierung für eine Immobilie abzuschließen. Gängig ist eine maximale Beleihung von höchstens $80 \%$ des Immobilienwerts. In vielen Ländern 

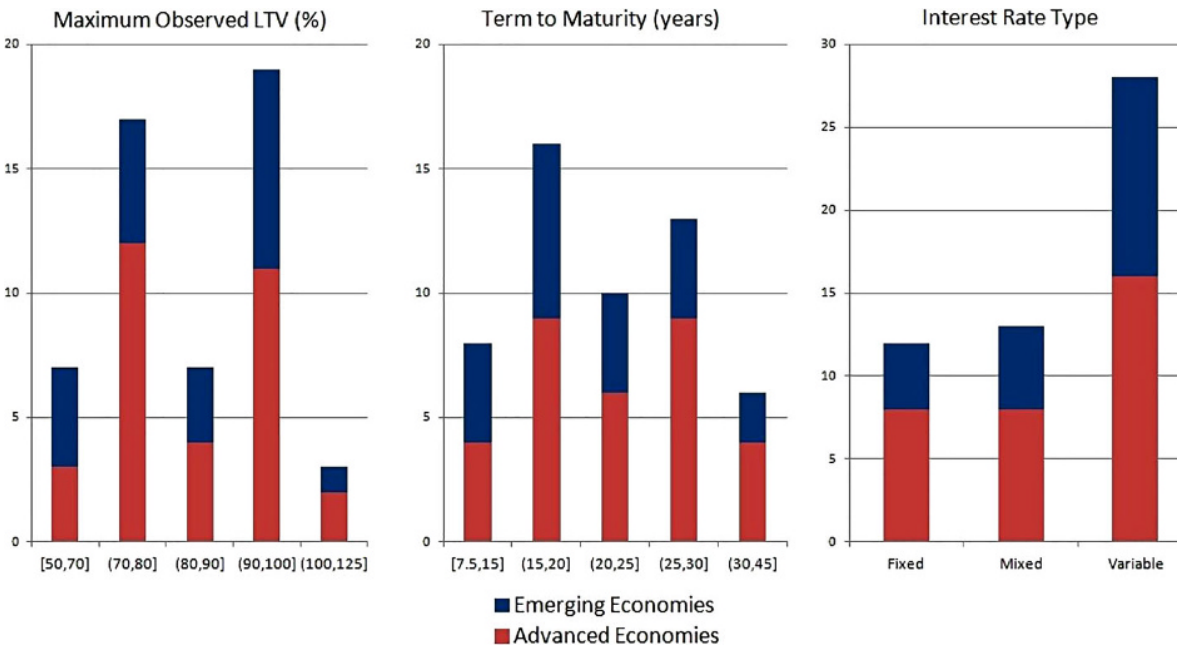

Abb. 3 Internationale Unterschiede: Kreditbetrag/Verkehrswert, Laufzeit und Art der Hypothek. (Quelle: Cerutti et al. 2015)

gelten höhere Beleihungsgrenzen, was insgesamt den Kreditzugang erleichtert (vgl. Abb. 3 und Cerutti et al. 2015).

\subsection{Besteuerung}

Die Besteuerung von Immobilientransaktionen schränkt die Liquidität von Wohnungsmärkten ein und macht den Erwerb von selbstgenutztem Eigentum unattraktiver. Hohe Grunderwerbsteuersätze erhöhen die Kosten der Transaktion und damit von Umzügen. Deutschlands Grunderwerbsteuersätze können seit dem Jahr 2007 durch die Länder festgelegt werden und sind seither von zunächst einheitlich 3,5\% auf mancherorts 6,5\% gestiegen (vgl. Abb. 4 und Bechtoldth et al. 2014).

\section{Der Einfluss von Erwartungen auf die Immobilienpreisbildung}

Die bis hierher genannten Faktoren mögen einen Teil der geringen Wohneigentumsquote in Deutschland erklären - häufig wird aber auch davon gesprochen, dass in Deutschland eine andere Einstellung gegenüber dem Wohneigentum herrscht. Dieser Aspekt wurde bislang empirisch kaum beleuchtet. Prinzipiell ist es denkbar, dass über den Wirkungskanal der Erwartungsbildung über die zukünftige Immobilienpreisentwicklung die Entscheidung für oder gegen den Kauf einer Immobilie getroffen wird. Dies leitet sich aus einer einfachen Berechnung des Gegenwartswerts ab: Wird eine feste Nutzungsdauer einer Immobilie angenommen, so ist ihr Gegenwartswert durch die möglichen Mietzahlungen in dieser Zeit zuzüglich dem Verkaufswert der Immobilie bestimmt. Unterscheiden sich die Erwartungen über den letztgenannten Aspekt zwischen einzelnen Bevölkerungsgruppen, dann dürfte auch deren Neigung Wohneigentum zu bilden unterschiedlich stark ausgeprägt sein. 


\begin{tabular}{|c|c|c|c|}
\hline \multirow{2}{*}{ Land } & \multirow{2}{*}{$\begin{array}{l}\text { Steuersatz } \\
\text { in Prozent }\end{array}$} & \multicolumn{2}{|c|}{ Variation des Steuersatzes } \\
\hline & & nach Immobilienwert & nach Region \\
\hline Argentinien & circa 3 & $x$ & $\checkmark$ \\
\hline Australien & $1,9-7,3$ & $\checkmark$ & $\checkmark$ \\
\hline Brasilien & circa 2 & $x$ & $\checkmark$ \\
\hline Bulgarien & $2-4$ & $x$ & $\checkmark$ \\
\hline China & 5,5 & $x$ & $x$ \\
\hline Dänemark & 0,6 & $x$ & $x$ \\
\hline Deutschland & $3,5-6,5$ & $x$ & $\checkmark$ \\
\hline Estland & keine & & \\
\hline Finnland & 4 & $x$ & $x$ \\
\hline Frankreich & 5,10 & $x$ & $x$ \\
\hline Griechenland & 3 & $x$ & $x$ \\
\hline Großbritannien & $0-4$ & $\checkmark$ & $x$ \\
\hline Indonesien & 5 & $x$ & $x$ \\
\hline Irland & $0-6$ & $\checkmark$ & $x$ \\
\hline Italien & $2-9$ & $x$ & $x$ \\
\hline Kroatien & 5 & $x$ & $x$ \\
\hline Lettland & 2 & $x$ & $x$ \\
\hline Litauen & keine & & \\
\hline Luxemburg & $6-9$ & $x$ & $\checkmark$ \\
\hline Niederlande & $2-6$ & $x$ & $x$ \\
\hline Österreich & 3,5 & $x$ & $x$ \\
\hline Polen & keine & & \\
\hline Russland & keine & & \\
\hline Schweden & 1,5 & $x$ & $x$ \\
\hline Schweiz & $1-3,3$ & $x$ & $\checkmark$ \\
\hline Slowakische Republik & keine & & \\
\hline Südafrika & $0-8$ & $\checkmark$ & $x$ \\
\hline Tschechische Republik & 4 & $x$ & $x$ \\
\hline Türkei & 2 & $x$ & $x$ \\
\hline Ungarn & $2-4$ & $x$ & $x$ \\
\hline USA & $0-2,6$ & $\checkmark$ & $\checkmark$ \\
\hline Vereinigte Arabische Emirate & 4 & $x$ & $x$ \\
\hline Zypern & $3-8$ & $\checkmark$ & $x$ \\
\hline
\end{tabular}

$\checkmark=J a, x=$ Nein.

Abb. 4 Besteuerung von Immobilientransaktionen im internationalen Vergleich. (Quelle: Bechtoldth et al. 2014) 
Diese Hypothese wird in einem einfachen empirischen Ansatz überprüft. Regressiert wird die erwartete (Miet-)Preisentwicklung auf zahlreiche sozio-ökonomische Variablen und eine Dummy-Variable, die für die deutsche Staatsbürgerschaft steht bzw. für die deutsche Staatsbürgerschaft und den Status als Mieter. Ein signifikanter Dummy dürfte, sofern das geschätzte Modell alle wichtigen Variablen enthält, den „kulturellen Hintergrund“ und Unterschiede zwischen der Bevölkerung deutscher Nationalität und der Bevölkerung aus allen anderen Ländern abbilden.

\subsection{Daten}

Die Studie verwendet Daten des SOEP-Innovation Samples für die Jahre 2016 und 2017. Hierbei handelt es sich um eine längsschnittliche Innovationsstichprobe bestehend aus neuen Surveydaten. Die Stichprobe erfragt unter anderem die Erwartungsbildung von Individuen in verschiedenen ökonomischen Bereichen wie zum Beispiel dem Immobilienmarkt. Explizit werden Miet- und Hauspreiserwartungen für die kommenden zwei Jahre und die nächsten 30 Jahre erfragt. Im Jahr 2016 nahmen 1556 Individuen an dem Immobilienmodul des SOEP-IS teil. Davon partizipierten 993 Individuen erneut im Jahr 2017. Zusätzlich nahmen 37 Individuen neu an der Befragung teil. Als Teil des Moduls beantworteten Teilnehmer unter anderem die folgenden Fragen:

- Was glauben Sie: um wie viel Prozent werden die Mieten in 2/30 Jahren über den heutigen Mieten liegen?

- Was glauben Sie: um wie viel Prozent wird der Kaufpreis in 2/30 Jahren über dem heutigen Kaufpreis liegen?

Insgesamt beantworten um die 2100 Individuen die Erwartungsfragen in der kurzen Frist. Die 30-jährigen Preiserwartungen wurden von ungefähr 1600 Individuen beantwortet.

\subsection{Regressionsanalyse}

Die folgenden Spezifikationen werden in einem Pooled OLS Model geschätzt, wobei die Standardfehler auf dem individuellen Level geclustered sind.

\section{Grundmodel:}

$$
P_{i}=\beta_{0}+\alpha_{i}+\beta_{1} \text { Deutsch }_{i}+X_{i}^{\prime} \beta_{2}+\beta_{3} T_{i}+u_{i}
$$

\section{Interaktionsmodel:}

$$
P_{i}=\beta_{0}+\alpha_{i}+\beta_{1} \text { Deutsch }_{i}+\beta_{2} \text { Mieter }_{i}+\beta_{3} \text { Mieter }_{i} * \operatorname{Deutsch}_{i}+X_{i}^{\prime} \beta_{4}+\beta_{5} T_{i}+u_{i}
$$

$P_{i}$ ist die abhängige Variable und stellt je nach Spezifikation die erfragten zwei Jahres- bzw. 30 Jahreserwartungen für Hauspreise und Mietpreise dar. Zusätzlich wird das Verhältnis der Hauspreis- und Mietpreiserwartungen gebildet und in einer 
separaten Schätzung der obigen Modelle als abhängige Variable verwendet. $\alpha_{i}$ sind regionale Fixeffekte auf der Ebene von Postleitregionen. Hierbei wird für die regionale Variation von Immobilienpreisen und Preiserwartungen kontrolliert. Postleitregionen werden durch die ersten zwei Ziffern der jeweiligen Postleitzahl festgelegt. Insgesamt gibt es in Deutschland 95 Postleitregionen. $X_{i}$ beinhaltet verschiedene demographische und sozio-ökonomische Kontrollvariablen, welche beispielsweise den individuellen Bildungsstand und Bruttoverdienst im Vormonat der Befragung messen. Zusätzlich kontrolliert $X_{i}$ für grobe Ausstattungsmerkmale der aktuell bewohnten Immobilie. So wird zum Beispiel für die Größe und die Art der Immobilie kontrolliert. $T_{i}$ ist ein Jahresindikator.

Die relevanten Variablen von Interesse sind die beiden Indikatoren Deutsch, Mieter $_{i}$ und die Interaktion aus beiden Variablen, Mieter $_{i} *$ Deutsch $_{i}$. Ziel der dargestellten Modelle ist es folglich:

1. $\mathrm{Zu}$ analysieren, ob deutsche Individuen signifikant geringere Haus- und Mietpreiserwartungen haben als Individuen nicht-deutscher Herkunft.

2. Zusätzlich soll mit Hilfe des Interaktionsmodells ergründet werden, ob deutsche Mieter signifikant unterschiedliche Preiserwartungen im Vergleich mit nicht-deutschen Mietern haben.

\subsection{Ergebnisse}

Die Ergebnisse über die Erwartungen zur Entwicklung der Häuserpreise sind in Tab. 1 zusammengefasst. Kontrolliert wird für demographische und sozio-ökonomische Faktoren sowie für die Ausstattung der aktuellen Wohnung und für regionale Fixeffekte auf der Ebene der Postleitregionen. Von Interesse sind in erster Linie die Ergebnisse der ersten drei Zeilen ${ }^{1}$.

Preiserwartungen Im Ergebnis zeigt sich, dass kurzfristige Unterschiede in den Erwartungen über die Immobilienpreisentwicklung existieren. Für den Horizont der kommenden beiden Jahre haben Befragte deutscher Nationalität signifikant geringere Preiserwartungen als die Kontrollgruppe mit internationalem Hintergrund. Dies gilt allerdings nur für die kurze Frist. Wird zusätzlich das Merkmal „Mieter“ in der Schätzgleichung berücksichtigt und mit der Nationalität interagiert, finden sich keine Unterschiede mehr in den Preiserwartungen, sowohl für den 2-Jahres-Horizont als auch für den Horizont von 30 Jahren.

Mieterwartungen Für die Mieterwartungen (vgl. Tab. 2) zeigt sich ein ähnliches Muster: in der kurzen Frist erwarten Befragte mit deutscher Nationalität einen deutlich schwächeren Mietanstieg als Personen anderer Nationalitäten. Dies zeigt, dass die Ertragserwartungen konsistent mit den Preiserwartungen verbunden sind.

\footnotetext{
1 Die Werte des $\mathrm{R}^{2}$ Terms liegen je nach Spezifikation zwischen 0,10 und 0,15. $\mathrm{R}^{2}$-Werte liegen in der Literatur zu Hauspreiserwartungen standartgemäß in dieser Höhe (vgl. Kuchler und Zafar 2015). Weitere Erwartungsvariablen wie zum Beispiel Zins- und Inflationserwartungen könnten zu höheren Werten des $\mathrm{R}^{2}$-Terms beitragen, werden jedoch in dieser Studie nicht beobachtet.
} 
Tab. 1 Hauspreiserwartungen

\begin{tabular}{|c|c|c|c|c|}
\hline & $\begin{array}{l}\text { I } \\
2 \text { Jahre }\end{array}$ & $\begin{array}{l}\text { II } \\
30 \text { Jahre }\end{array}$ & $\begin{array}{l}\text { III } \\
2 \text { Jahre }\end{array}$ & $\begin{array}{l}\text { IV } \\
30 \text { Jahre }\end{array}$ \\
\hline$\overline{\text { Deutsch }}$ & $\begin{array}{l}-5,122 * * \\
(2,041)\end{array}$ & $\begin{array}{l}-16,639 \\
(12,629)\end{array}$ & $\begin{array}{l}-3,366 \\
(2,455)\end{array}$ & $\begin{array}{l}-5,945 \\
(5,795)\end{array}$ \\
\hline Mieter & $\begin{array}{l}-0,322 \\
(1,069)\end{array}$ & $\begin{array}{l}2,424 \\
(3,380)\end{array}$ & $\begin{array}{l}3,154 \\
(4,205)\end{array}$ & $\begin{array}{l}21,739 \\
(21,344)\end{array}$ \\
\hline Deutsche Mieter & - & - & $\begin{array}{l}-3,742 \\
(4,136)\end{array}$ & $\begin{array}{l}-21,090 \\
(22,914)\end{array}$ \\
\hline \multicolumn{5}{|c|}{ Demographische und sozio-ökonomische Kontrollvariablen } \\
\hline Vollzeit & $\begin{array}{l}1,510 \\
(1,040)\end{array}$ & $\begin{array}{l}-0,837 \\
(5,529)\end{array}$ & $\begin{array}{l}1,513 \\
(1,041)\end{array}$ & $\begin{array}{l}-0,899 \\
(5,466)\end{array}$ \\
\hline Weiblich & $\begin{array}{l}-0,332 \\
(0,723)\end{array}$ & $\begin{array}{l}-12,318 * * * \\
(2,943)\end{array}$ & $\begin{array}{l}-0,281 \\
(0,732)\end{array}$ & $\begin{array}{l}-12,059 * * * \\
(2,956)\end{array}$ \\
\hline Alter & $\begin{array}{l}0,030 \\
(0,025)\end{array}$ & $\begin{array}{l}-0,013 \\
(0,075)\end{array}$ & $\begin{array}{l}0,030 \\
(0,025)\end{array}$ & $\begin{array}{l}0,125 \\
(0,075)\end{array}$ \\
\hline Bruttoverdienst Vormonat & $\begin{array}{l}-0,00069^{* *} \\
(0,000)\end{array}$ & $\begin{array}{l}0,00052 \\
(0,001)\end{array}$ & $\begin{array}{l}-0,00068^{* *} \\
(0,000)\end{array}$ & $\begin{array}{l}0,00060 \\
(0,001)\end{array}$ \\
\hline Verheiratet & $\begin{array}{l}0,026 \\
(0,669)\end{array}$ & $\begin{array}{l}-7,093 \\
(3,495)\end{array}$ & $\begin{array}{l}-0,003 \\
(0,665)\end{array}$ & $\begin{array}{l}-7,414 * * \\
(3,634)\end{array}$ \\
\hline Abitur & $\begin{array}{l}-0,300 \\
(0,818)\end{array}$ & $\begin{array}{l}8,840 * * * \\
(3,207)\end{array}$ & $\begin{array}{l}-0,334 \\
(0,820)\end{array}$ & $\begin{array}{l}8,570 * * * \\
(3,305)\end{array}$ \\
\hline $\begin{array}{l}\text { Zufriedenheit mit } \\
\text { Immobilie }\end{array}$ & $\begin{array}{l}-0,186 \\
(0,284)\end{array}$ & $\begin{array}{l}-0,090 \\
(1,306)\end{array}$ & $\begin{array}{l}-0,175 \\
(0,284)\end{array}$ & $\begin{array}{l}-0,027 \\
(1,249)\end{array}$ \\
\hline Risikobereitschaft & $\begin{array}{l}-0,021 \\
(0,132)\end{array}$ & $\begin{array}{l}-1,154 \\
(0,712)\end{array}$ & $\begin{array}{l}-0,021 \\
(0,132)\end{array}$ & $\begin{array}{l}-1,176 \\
(0,698)\end{array}$ \\
\hline \multicolumn{5}{|l|}{ Ausstattungsmerkmale } \\
\hline Wohnfläche in Qm & $\begin{array}{l}-0,006 \\
(0,009)\end{array}$ & $\begin{array}{l}-0,079 * \\
(0,044)\end{array}$ & $\begin{array}{l}-0,006 \\
(0,009)\end{array}$ & $\begin{array}{l}-0,082 * \\
(0,043)\end{array}$ \\
\hline Wohnhaus & $\begin{array}{l}-0,797 \\
(1,604)\end{array}$ & $\begin{array}{l}-0,599 \\
(7,413)\end{array}$ & $\begin{array}{l}-0,748 \\
(1,607)\end{array}$ & $\begin{array}{l}-0,392 \\
(7,534)\end{array}$ \\
\hline Ein-/Zweifamilienhaus & $\begin{array}{l}-1,133 \\
(1,166)\end{array}$ & $\begin{array}{l}-2,618 \\
(3,231)\end{array}$ & $\begin{array}{l}-1,166 \\
(1,162)\end{array}$ & $\begin{array}{l}-2,835 \\
(3,181)\end{array}$ \\
\hline$J a h r=2017$ & $\begin{array}{l}0,579 \\
(0,980)\end{array}$ & $\begin{array}{l}2,101 \\
(2,664)\end{array}$ & $\begin{array}{l}0,582 \\
(0,980)\end{array}$ & $\begin{array}{l}2,069 \\
(2,663)\end{array}$ \\
\hline Konstante & $\begin{array}{l}16,580 * * * \\
(3,381)\end{array}$ & $\begin{array}{l}60,842 * * * \\
(19,557)\end{array}$ & $\begin{array}{l}14,824 * * * \\
(3,842)\end{array}$ & $\begin{array}{l}50,819 * * * \\
(13,128)\end{array}$ \\
\hline Regionale Fixeffekte & $\mathrm{x}$ & $\mathrm{x}$ & $\mathrm{x}$ & $\mathrm{x}$ \\
\hline$R 2$ & 0,108 & 0,144 & 0,109 & 0,146 \\
\hline$N$ & 1889 & 1460 & 1889 & 1460 \\
\hline
\end{tabular}

$* p \leq 0,10 ; * * p \leq 0,05 ; * * * p \leq 0,010$ 
Tab. 2 Mietpreiserwartungen

\begin{tabular}{|c|c|c|c|c|}
\hline & $\begin{array}{l}\text { I } \\
2 \text { Jahre }\end{array}$ & $\begin{array}{l}\text { II } \\
30 \text { Jahre }\end{array}$ & $\begin{array}{l}\text { III } \\
2 \text { Jahre }\end{array}$ & $\begin{array}{l}\text { IV } \\
30 \text { Jahre }\end{array}$ \\
\hline Deutsch & $\begin{array}{l}-4,722 * * * \\
(1,673)\end{array}$ & $\begin{array}{l}-1,060 \\
(5,675)\end{array}$ & $\begin{array}{l}-2,470 \\
(2,172)\end{array}$ & $\begin{array}{l}-1,948 \\
(5,897)\end{array}$ \\
\hline Mieter & $\begin{array}{l}0,676 \\
(0,631)\end{array}$ & $\begin{array}{l}1,118 \\
(2,832)\end{array}$ & $\begin{array}{l}4,915 \\
(3,531)\end{array}$ & $\begin{array}{l}-0,494 \\
(10,027)\end{array}$ \\
\hline Deutsche Mieter & - & - & $\begin{array}{l}-4,580 \\
(3,428)\end{array}$ & $\begin{array}{l}1,748 \\
(10,366)\end{array}$ \\
\hline \multicolumn{5}{|c|}{ Demographische und sozio-ökonomische Kontrollvariablen } \\
\hline Vollzeit & $\begin{array}{l}0,424 \\
(0,706)\end{array}$ & $\begin{array}{l}-7,042 \\
(4,892)\end{array}$ & $\begin{array}{l}0,390 \\
(0,708)\end{array}$ & $\begin{array}{l}-7,030 \\
(4,886)\end{array}$ \\
\hline Weiblich & $\begin{array}{l}0,534 \\
(0,468)\end{array}$ & $\begin{array}{l}-11,280 * * * \\
(2,790)\end{array}$ & $\begin{array}{l}0,576 \\
(0,479)\end{array}$ & $\begin{array}{l}-11,295 * * * \\
(2,815)\end{array}$ \\
\hline Alter & $\begin{array}{l}-0,026^{*} \\
(0,015 \mathrm{~s})\end{array}$ & $\begin{array}{l}0,004 \\
(0,095)\end{array}$ & $\begin{array}{l}-0,027 * \\
(0,015)\end{array}$ & $\begin{array}{l}0,004 \\
(0,095)\end{array}$ \\
\hline Bruttoverdienst Vormonat & $\begin{array}{l}-0,000^{* *} \\
(0,000)\end{array}$ & $\begin{array}{l}0,002 * \\
(0,001)\end{array}$ & $\begin{array}{l}-0,000 * * \\
(0,000)\end{array}$ & $\begin{array}{l}0,002 * * \\
(0,001)\end{array}$ \\
\hline Verheiratet & $\begin{array}{l}0,564 \\
(0,488)\end{array}$ & $\begin{array}{l}-0,505 \\
(2,479)\end{array}$ & $\begin{array}{l}0,515 \\
(0,476)\end{array}$ & $\begin{array}{l}-0,482 \\
(2,470)\end{array}$ \\
\hline Abitur & $\begin{array}{l}-0,361 \\
(0,447)\end{array}$ & $\begin{array}{l}7,563 * * \\
(3,573)\end{array}$ & $\begin{array}{l}0,412 \\
(0,447)\end{array}$ & $\begin{array}{l}7,582 * * \\
(3,572)\end{array}$ \\
\hline $\begin{array}{l}\text { Zufriedenheit mit } \\
\text { Immobilie }\end{array}$ & $\begin{array}{l}0,039 \\
(0,134)\end{array}$ & $\begin{array}{l}0,095 \\
(0,632)\end{array}$ & $\begin{array}{l}0,051 \\
(0,134)\end{array}$ & $\begin{array}{l}0,088 \\
(0,633)\end{array}$ \\
\hline Risikobereitschaft & $\begin{array}{l}0,027 \\
(0,081)\end{array}$ & $\begin{array}{l}1,023 * * \\
(0,468)\end{array}$ & $\begin{array}{l}0,027 \\
(0,081)\end{array}$ & $\begin{array}{l}-1,020 * * \\
(0,468)\end{array}$ \\
\hline \multicolumn{5}{|l|}{ Ausstattungsmerkmale } \\
\hline Wohnfläche in Qm & $\begin{array}{l}-0,011 * \\
(0,006)\end{array}$ & $\begin{array}{l}-0,076^{* * *} \\
(0,026)\end{array}$ & $\begin{array}{l}-0,011^{*} \\
(0,006)\end{array}$ & $\begin{array}{l}-0,076^{* * *} \\
(0,026)\end{array}$ \\
\hline Wohnhaus & $\begin{array}{l}0,473 \\
(0,647)\end{array}$ & $\begin{array}{l}4,412 \\
(4,236)\end{array}$ & $\begin{array}{l}0,508 \\
(0,647)\end{array}$ & $\begin{array}{l}4,390 \\
(4,221)\end{array}$ \\
\hline $\begin{array}{l}\text { Dummy Ein-/ } \\
\text { Zweifamilienhaus }\end{array}$ & $\begin{array}{l}0,671 \\
(0,734)\end{array}$ & $\begin{array}{l}0,005 \\
(2,680)\end{array}$ & $\begin{array}{l}0,606 \\
(0,726)\end{array}$ & $\begin{array}{l}0,023 \\
(2,690)\end{array}$ \\
\hline$J a h r=2017$ & $\begin{array}{l}0,428 \\
(0,401)\end{array}$ & $\begin{array}{l}1,646 \\
(3,006)\end{array}$ & $\begin{array}{l}0,443 \\
(0,399)\end{array}$ & $\begin{array}{l}1,649 \\
(3,009)\end{array}$ \\
\hline Konstante & $\begin{array}{l}13,996 * * * \\
(2,496)\end{array}$ & $\begin{array}{l}43,680 * * * \\
(9,563)\end{array}$ & $\begin{array}{l}11,821 * * * \\
(3,137)\end{array}$ & $\begin{array}{l}44,535 * * * \\
(10,628)\end{array}$ \\
\hline Regionale Fixeffekte & $\mathrm{x}$ & $\mathrm{x}$ & $\mathrm{x}$ & $\mathrm{x}$ \\
\hline$R 2$ & 0,115 & 0,158 & 0,119 & 0,158 \\
\hline$N$ & 1938 & 1486 & 1938 & 1486 \\
\hline
\end{tabular}

$* p \leq 0,10 ; * * p \leq 0,05 ; * * * p \leq 0,010$ 
Tab. 3 Verhältnis aus Haus- und Mietpreiserwartungen

\begin{tabular}{|c|c|c|c|c|}
\hline & $\begin{array}{l}\text { I } \\
2 \text { Jahre }\end{array}$ & $\begin{array}{l}\text { II } \\
30 \text { Jahre }\end{array}$ & $\begin{array}{l}\text { III } \\
2 \text { Jahre }\end{array}$ & $\begin{array}{l}\text { IV } \\
30 \text { Jahre }\end{array}$ \\
\hline$\overline{\text { Deutsch }}$ & $\begin{array}{l}0,213 \\
(0,266)\end{array}$ & $\begin{array}{l}-0,143 \\
(0,270)\end{array}$ & $\begin{array}{l}0,480 \\
(0,437)\end{array}$ & $\begin{array}{l}-0,013 \\
(0,468)\end{array}$ \\
\hline Mieter & $\begin{array}{l}0,004 \\
(0,158)\end{array}$ & $\begin{array}{l}0,181 \\
(0,199)\end{array}$ & $\begin{array}{l}0,514 \\
(0,446)\end{array}$ & $\begin{array}{l}-0,013 \\
(0,570)\end{array}$ \\
\hline Deutscher Mieter & - & - & $\begin{array}{l}0,557 \\
(0,462)\end{array}$ & $\begin{array}{l}-0,183 \\
(0,630)\end{array}$ \\
\hline \multicolumn{5}{|c|}{ Demographische und sozio-ökonomische Kontrollvariablen } \\
\hline Vollzeit & $\begin{array}{l}-0,029 \\
(0,156)\end{array}$ & $\begin{array}{l}0,105 \\
(0,139)\end{array}$ & $\begin{array}{l}-0,031 \\
(0,157)\end{array}$ & $\begin{array}{l}0,103 \\
(0,139)\end{array}$ \\
\hline Weiblich & $\begin{array}{l}-0,206 \\
(0,165)\end{array}$ & $\begin{array}{l}-0,080 \\
(0,107)\end{array}$ & $\begin{array}{l}-0,200 \\
(0,164)\end{array}$ & $\begin{array}{l}-0,078 \\
(0,108)\end{array}$ \\
\hline Alter & $\begin{array}{l}0,010 * * * \\
(0,004)\end{array}$ & $\begin{array}{l}0,003 \\
(0,005)\end{array}$ & $\begin{array}{l}0,010 * * * \\
(0,004)\end{array}$ & $\begin{array}{l}0,003 \\
(0,005)\end{array}$ \\
\hline Bruttoverdienst Vormonat & $\begin{array}{l}-0,000 \\
(0,000)\end{array}$ & $\begin{array}{l}-0,000 \\
(0,000)\end{array}$ & $\begin{array}{l}-0,000 \\
(0,000)\end{array}$ & $\begin{array}{l}-0,000 \\
(0,000)\end{array}$ \\
\hline Verheiratet & $\begin{array}{l}0,062 \\
(0,138)\end{array}$ & $\begin{array}{l}-0,101 \\
(0,187)\end{array}$ & $\begin{array}{l}-0,066 \\
(0,138)\end{array}$ & $\begin{array}{l}-0,103 \\
(0,189)\end{array}$ \\
\hline Abitur & $\begin{array}{l}-0,057 \\
(0,141)\end{array}$ & $\begin{array}{l}-0,214 \\
(0,135)\end{array}$ & $\begin{array}{l}-0,062 \\
(0,143)\end{array}$ & $\begin{array}{l}-0,216 \\
(0,138)\end{array}$ \\
\hline $\begin{array}{l}\text { Zufriedenheit mit } \\
\text { Immobilie }\end{array}$ & $\begin{array}{l}0,019 \\
(0,035)\end{array}$ & $\begin{array}{l}-0,009 \\
(0,033)\end{array}$ & $\begin{array}{l}0,021 \\
(0,035)\end{array}$ & $\begin{array}{l}-0,009 \\
(0,032)\end{array}$ \\
\hline Risikobereitschaft & $\begin{array}{l}-0,012 \\
(0,028)\end{array}$ & $\begin{array}{l}0,022 \\
(0,032)\end{array}$ & $\begin{array}{l}-0,012 \\
(0,028)\end{array}$ & $\begin{array}{l}0,022 \\
(0,032)\end{array}$ \\
\hline \multicolumn{5}{|l|}{ Ausstattungsmerkmale } \\
\hline Wohnfläche in QM & $\begin{array}{l}0,002 \\
(0,002)\end{array}$ & $\begin{array}{l}0,002 \\
(0,002)\end{array}$ & $\begin{array}{l}0,002 \\
(0,002)\end{array}$ & $\begin{array}{l}0,002 \\
(0,002)\end{array}$ \\
\hline Wohnhaus & $\begin{array}{l}-0,292 \\
(0,182)\end{array}$ & $\begin{array}{l}-0,125 \\
(0,168)\end{array}$ & $\begin{array}{l}-0,286 \\
(0,182)\end{array}$ & $\begin{array}{l}-0,122 \\
(0,171)\end{array}$ \\
\hline Ein-/Zweifamilienhaus & $\begin{array}{l}-0,318 \\
(0,234)\end{array}$ & $\begin{array}{l}-0,176 \\
(0,182)\end{array}$ & $\begin{array}{l}-0,324 \\
(0,236)\end{array}$ & $\begin{array}{l}-0,177 \\
(0,183)\end{array}$ \\
\hline$J a h r=2017$ & $\begin{array}{l}-0,031 \\
(0,158)\end{array}$ & $\begin{array}{l}-0,095 \\
(0,130)\end{array}$ & $\begin{array}{l}-0,030 \\
(0,158)\end{array}$ & $\begin{array}{l}-0,095 \\
(0,130)\end{array}$ \\
\hline Konstante & $\begin{array}{l}0,824 * \\
(0,446)\end{array}$ & $\begin{array}{l}1,180 * * \\
(0,474)\end{array}$ & $\begin{array}{l}0,557 \\
(0,569)\end{array}$ & $\begin{array}{l}1,090 * \\
(0,644)\end{array}$ \\
\hline Regionale Fixeffekte & $\mathrm{x}$ & $\mathrm{x}$ & $\mathrm{x}$ & $\mathrm{x}$ \\
\hline$R 2$ & 0,063 & 0,058 & 0,064 & 0,058 \\
\hline$N$ & 1794 & 1340 & 1794 & 1340 \\
\hline
\end{tabular}

$* p \leq 0,10 ; * * p \leq 0,05 ; * * * p \leq 0,010$ 
Geringere Erträge führen in der Investitionsrechnung zu geringeren Zahlungsbereitschaften, d.h. zu niedrigeren Preiserwartungen. Dieses Ergebnis hält ebenfalls nur für den Fall, in dem nicht die Nationalität mit dem Status der Mieterhaushalte interagiert wird. Wird hierfür kontrolliert, stimmen zwar nach wie vor die Vorzeichen, allerdings sind die Schätzungen nicht mehr statistisch von null verschieden.

Preis-Miet-Verhältnis Wenn Ertrags- und Erlöserwartungen im Einklang stehen, dürfte dies zu keinen Unterschieden in deren Verhältnissen führen. Dies ist gewissermaßen der Test für die Rationalität der Preiserwartungen. Regressiert wird daher das Verhältnis dieser beiden Größen auf die bereits bekannten Kontrollvariablen und das Merkmal der Nationalität. Die Ergebnisse in Tab. 3 bestätigen diese Annahme: Es zeigt sich weder ein Einfluss der Nationalität noch die Interaktion aus Nationalität und dem Status als Mieter auf das Verhältnis von Preisen und Mieten.

\section{Schlussfolgerungen}

Die Diskussion über die niedrige Einkommensquote in Deutschland wird derzeit politisch intensiv geführt. Die derzeitige Regierung hat unterschiedliche Maßnahmen beschlossen, um die Eigentumsquote zu erhöhen und die Ersparnisbildung in Immobilienvermögen zu stärken. Prominentes Beispiel sind das Baukindergeld, die Wohnungsbauprämie, die Diskussion um die Grunderwerbsteuer und Initiativen zur Stärkung des Eigenkapitals für den Immobilienerwerb. Prinzipiell setzt die Politik an einigen auch in der Literatur identifizierten Stellschrauben an.

Das vorliegende Papier hat einen weiteren Aspekt beleuchtet, der zwar immer wieder genannt wird, aber bislang nicht empirisch untersucht wurde. Auch die Einstellungen und Erwartungen von Haushalten über die zukünftige Marktentwicklung können einen erheblichen Einfluss auf das Marktgeschehen ausüben. Sind bestimmte Haushaltsgruppen prinzipiell pessimistischer, dann dürften diese eine weniger ausgeprägte Neigung zur Bildung von Immobilienvermögen haben. Erste Anzeichen hierfür finden sich in den Daten: Es zeigt sich, dass befragte Personen mit deutschem Pass signifikant geringere Erwartungen an die kurzfristige Preisentwicklung haben als Personen mit anderer Nationalität. Die Preiserwartungen scheinen sich dabei aus einer deutlich pessimistischeren Einschätzung der Ertragsentwicklung zu speisen. Auch die Erwartung zur Mietdynamik ist deutlich schwächer bei deutschen Befragten. Unabhängig von der Treffgenauigkeit der Erwartungen zeigt dies, dass Personen ihre Erwartungen an die Marktentwicklung knüpfen und offenbar keinen spekulativen Motiven folgen. Dies zeigt sich in der Analyse des KaufpreisMietverhältnisses, bei dem keine Unterschiede zwischen den Bevölkerungsgruppen nachweisbar sind.

Förderung Dieses Projekt wurde durch die Deutsche Forschungsgemeinschaft finanziell unterstützt (CRC TRR 190).

We gratefully acknowledge financial support by the German Science Foundation through CRC TRR 190. 


\section{Anhang}

\section{Deskriptive Ergebnisse}

Tab. 4 Zusammenfassende Statistiken 2016-2017

Tab. 5 Zusammenfassende Statistiken 2016

Tab. 6 Zusammenfassende Statistiken 2017

\begin{tabular}{lccll}
\hline \multicolumn{3}{c}{ Mietpreise } & \multicolumn{2}{c}{ Hauspreise } \\
& 2 Jahre & 30 Jahre & 2 Jahre & 30 Jahre \\
\hline Durchschnittliche & Erwartungen & & \\
Gesamt & 8,31 & 29,56 & 9,74 & 30,14 \\
Deutsch & 8,05 & 29,45 & 9,53 & 29,34 \\
Nicht-Deutsch & 11,13 & 31,14 & 12,20 & 39,16 \\
Beobachtungen & & & & \\
Gesamt & 2170 & 1644 & 2103 & 1611 \\
Deutsch & 1988 & 1502 & 1937 & 1478 \\
Nicht-Deutsch & 180 & 140 & 164 & 131 \\
\hline
\end{tabular}

\begin{tabular}{|c|c|c|c|c|}
\hline & \multicolumn{2}{|c|}{ Mietpreise } & \multicolumn{2}{|c|}{ Hauspreise } \\
\hline & 2 Jahre & 30 Jahre & 2 Jahre & 30 Jahre \\
\hline \multicolumn{5}{|c|}{ Durchschnittliche Erwartungen } \\
\hline Gesamt & 8,31 & 28,67 & 9,79 & 29,18 \\
\hline Deutsch & 7,87 & 28,54 & 9,32 & 28,07 \\
\hline Nicht-Deutsch & 13,76 & 30,21 & 15,63 & 42,92 \\
\hline \multicolumn{5}{|l|}{ Beobachtungen } \\
\hline Gesamt & 1293 & 1044 & 1253 & 1017 \\
\hline Deutsch & 1195 & 964 & 1159 & 941 \\
\hline Nicht-Deutsch & 98 & 80 & 94 & 76 \\
\hline
\end{tabular}

\begin{tabular}{lllll}
\hline \multicolumn{3}{c}{$\begin{array}{l}\text { Mietpreise } \\
\text { 2 Jahre }\end{array}$} & 30 Jahre & Hauspreise \\
2 Jahre & 30 Jahre \\
\hline Durchschnittliche & Erwartungen & & \\
Gesamt & 8,29 & 31,10 & 9,67 & 31,77 \\
Deutsch & 8,33 & 31,07 & 9,84 & 31,56 \\
Nicht-Deutsch & 8 & 32,38 & 7,6 & 33,96 \\
Beobachtungen & & & & \\
Gesamt & 877 & 600 & 850 & 1017 \\
Deutsch & 793 & 538 & 778 & 537 \\
Nicht-Deutsch & 98 & 80 & 94 & 76 \\
\hline
\end{tabular}


Open Access Dieser Artikel wird unter der Creative Commons Namensnennung 4.0 International Lizenz (http://creativecommons.org/licenses/by/4.0/deed.de) veröffentlicht, welche die Nutzung, Vervielfältigung, Bearbeitung, Verbreitung und Wiedergabe in jeglichem Medium und Format erlaubt, sofern Sie den/die ursprünglichen Autor(en) und die Quelle ordnungsgemäß nennen, einen Link zur Creative Commons Lizenz beifügen und angeben, ob Änderungen vorgenommen wurden.

\section{Literatur}

Bechtoldth S, Freier R, Geyer J, Kühn F (2014) Acht Jahre nach der Reform der Grunderwerbsteuer: Bundesländer nutzen ihre Spielräume für Steuererhöhungen. DIW Wochenber 81(50):1283-1290

Cerutti E, Dagher J, Dell'Ariccia G (2015) Housing finance and real-estate booms: a cross-country perspective. International Monetary Fund staff discussion note, Bd. 15/12

Chiuri MC, Jappelli T (2003) Financial market imperfections and home ownership: a comparative study. Eur Econ Rev 47(5):857-875

Chiuri MC, Jappelli T (2010) Do the elderly reduce housing equity? An international comparison. J Popul Econ 23(2):643-663

Clark WAV, Deurloo MC, Dieleman FM (1997) Entry to home-ownership in Germany: some comparisons with the United States. Urban Stud 34(1):7-19

Diaz-Serrano L (2009) Disentangling the housing satisfaction puzzle: Does homeownership really matter? J Econ Psychol 30(5):745-755

Fisher LM, Jaffe AJ (2003) Determinants of international home ownership rates. Hous Finance Int 18(1):34-37

Haurin DR, Dietz RD, Weinberg BA (2002) The impact of neighborhood homeownership rates: a review of the theoretical and empirical literature. J Hous Res 13(2):119

Henretta JC (1984) Parental status and child's home ownership. Am Sociol Rev 49(1):131-140

Holmqvist E, Turner LM (2014) Swedish welfare state and housing markets: under economic and political pressure. J Hous Built Environ 29(2):237-254

Kuchler T, Zafar B (2015) Personal experience and expectations about aggregate outcomes. Federal Reserve Bank of New York staff reports, Bd. 748

Leifels A, Raffelhüschen B (2014) Altersvorsorge durch Wohneigentum. In: Voigtländer M, Dependeuer O (Hrsg) Wohneigentum. Herausforderungen und Perspektiven. Bibliothek des Eigentums, Bd. 11. Springer, Heidelberg, S 129-155

Lerbs OW, Oberst CA (2014) Explaining the spatial variation in homeownership rates: results for German regions. Reg Stud 48(5):844-865

Michelsen C (2017, 6. Sept). Erwerb von Wohneigentum: Eigenkapitalschwelle für immer mehr Haushalte zu hoch. DIW-Aktuell 2

Michelsen C, Bach S, Harnisch M (2018, 11. Juli) Baukindergeld: Einkommensstarke Haushalte profitieren in besonderem Maße. DIW-Aktuell 14

Mulder CH, Wagner M (1998) First-time home-ownership in the family life course: a West German-Dutch comparison. Urban Stud 35(4):687-713

Rohe WM, Lindblad M (2013) Reexamining the social benefits of homeownership after the housing crisis. Joint Center for Housing Studies of Harvard University, Boston

Sommer K, Sullivan P (2018) Implications of us tax policy for house prices, rents, and homeownership. Am Econ Rev 108(2):241-274

SVR Wirtschaft (2016) Zeit für Reformen. Jahresgutachten 2016/17. Technical report. Sachverständigenrat zur Begutachtung der Gesamtwirtschaftlichen Entwicklung, Wiesbaden

Turner TM, Luea H (2009) Homeownership, wealth accumulation and income status. J Hous Econ 18(2):104-114

Voigtländer M (2009) Why is the German homeownership rate so low? Hous Stud 24(3):355-372 